\title{
Photoswitchable molecular dipole antennas with tailored coherent coupling in glassy composite
}

\author{
Mady Elbahri ${ }^{1,2}$, Ahnaf Usman Zillohu ${ }^{1}$, Bastian Gothe ${ }^{1}$, Mehdi Keshavarz Hedayati ${ }^{1}$, Ramzy Abdelaziz ${ }^{1,3}$, Hala $^{1}$ \\ Jarallah El-Khozondar ${ }^{4}$, Muhammad Bawa'aneh ${ }^{5}$, Moheb Abdelaziz ${ }^{1}$, Andrei Lavrinenko ${ }^{6}$, Sergei Zhukovsky ${ }^{6}$ \\ and Shahin Homaeigohar ${ }^{2}$
}

Here, we introduce the first experimental proof of coherent oscillation and coupling of photoswitchable molecules embedded randomly in a polymeric matrix and acting cooperatively upon illumination with UV light. In particular, we demonstrate the specular reflection and Brewster phenomenon alteration of photochromic molecular dipole antennas. We successfully demonstrate the concept of Brewster wavelength, which is based on the dipolar interaction between radiating dipoles and the surrounding matrix possessing a net dipole moment, as a key tool for highly localized sensing of matrix polarity. We also introduce the concept of "tailored molecular photonic coupling' while highlighting the role of interferences for the design of optically active media by adjusting the photonic response of the medium with the real and imaginary refractive index of photoswitchable molecules in the 'ON' state. Our results enhance our fundamental understanding of coherent dipole radiation and open a new vein of research based on glassy disordered dipolar composites that act as macroscopic antenna with cooperative action; furthermore, these results have important implications for new design rules of tailored photonics.

Light: Science \& Applications (2015) 4, e316; doi:10.1038/Isa.2015.89; published online 31 July 2015

Keywords: Brewster angle; coherent coupling; collective oscillation; photoswitchable molecular photonics; specular reflection

\section{INTRODUCTION}

Photoswitching is a highly desirable feature for modern applications ${ }^{1}$ ranging from memory devices ${ }^{2}$ and smart windows ${ }^{3}$ to optoelectronic and chemo-mechanical devices ${ }^{4}$. Photochromic molecules incorporated in a polymeric matrix compose a versatile system that has been investigated and developed for the construction of novel dynamic materials ${ }^{5,6}$ wherein the desired properties are not only tunable but also reversible ${ }^{7}$. Most of the progress is solely based on the optical absorption of the molecules. Despite its wide range of applications, some fundamental aspects and properties are still unclear or unexplored, for example, photoswitchable molecular reflection, which would open up entirely new possibilities in the ever advancing fields of smart and active optical sensing, photonics and plasmonics. For example, the common wisdom is that the change of optical properties of photoswitchable molecules originates from electronic transition; however, we believe that the roles of electronic polarization and dipole radiations have been overlooked. Here, we aim to introduce the concept of molecular dipole oscillation, cooperative coupling and coherent radiation of molecular dipole antennas. We demonstrate the first experimental proof of specular reflection at visible frequencies, which originates from and is controlled by photochromic molecular dipoles embedded randomly in a polymeric matrix under dynamic light switching. We introduce the Brewster wavelength concept and a new type of remote polarity sensor based on the dipole moment of the surrounding medium. By tailoring the specular reflection, interferences, absorption and refractive index modulation, we further demonstrate the design of molecular photonic coupling and introduce a new class of optically active photoswitchable devices.

\section{MATERIALS AND METHODS}

For the spin coating of films, solutions of a polymer ( $2 \mathrm{wt}-\%$ polystyrene (PS), $3 \mathrm{wt}-\%$ polyethersulfone and $2 \mathrm{wt}-\%$ polyvinylidene fluoride [PVDF]) together with 1,3-Dihydro-1,3,3-trimethylspiro[2H-indole$2,3^{\prime}$ - $[3 \mathrm{H}]$ phenanthr[ $[9,10-\mathrm{b}](1,4)$ oxazine $](\mathrm{SPO})$ were prepared by stirring in $\mathrm{N}, \mathrm{N}$-dimethylformamide (DMF) at room temperature. Glass slides were cleaned in piranha solution (volume ratio: $5 \mathrm{H}_{2} \mathrm{O}$ $+1 \mathrm{NH}_{4} \mathrm{OH}+1 \mathrm{H}_{2} \mathrm{O}_{2}$ ), rinsed with de-ionized water and subsequently dried with nitrogen. The spinning speed ranged from 2000 to $3600 \mathrm{r} \mathrm{min}^{-1}$. Film thickness was determined with a Bruker Dektak XT profilometer.

Solutions for electrospinning were prepared by dissolving 10-13 wt-\% PVDF in DMF by stirring along with SPO. Electrospinning was carried out by pushing the polymer solution at a fixed rate through a needle that was kept at a high potential with respect to a

${ }^{1}$ Nanochemistry and Nanoengineering, Faculty of Engineering, Institute for Materials Science, University of Kiel, Kaiserstrasse 2 , 24143 Kiel, Germany; ${ }^{2}$ Nanochemistry and

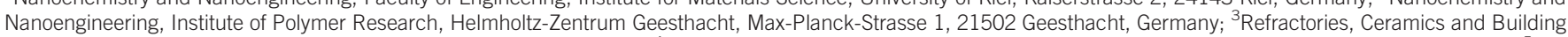

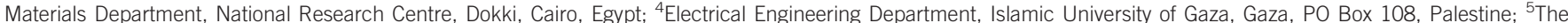

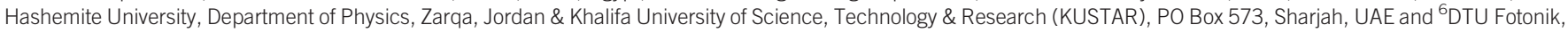
Technical University of Denmark, Oersteds pl. 343, Kongens Lyngby, DK-2800, Denmark 
grounded target in the form of two parallel wires. Because of electrostatic forces between the charged polymer and the grounded target, the polymer droplet formed a jet that further thinned into nanofibers during its flight toward the target. The fibers were collected between the two wires constituting the target and were placed onto a glass plate.

For switching SPO between its 'ON' and 'OFF' states, a UV lamp (Labino, $35 \mathrm{~W}$; Solna, Sweden) with a peak intensity at $365 \mathrm{~nm}$ was used with an irradiation time of approximately 4 minutes.

Ellipsometry was performed on an M-2000 ellipsometer from J.A. Woollam Co., Inc. (Lincoln, America) with an automated angle base. Measurements were conducted between $45^{\circ}$ and $85^{\circ}$ with a step size of $10^{\circ}$. Data acquisition was performed with the company's CompleteEASE software. UV-VIS analysis of films and fibers was performed using a PerkinElmer UV-VIS spectrometer (Lambda

a

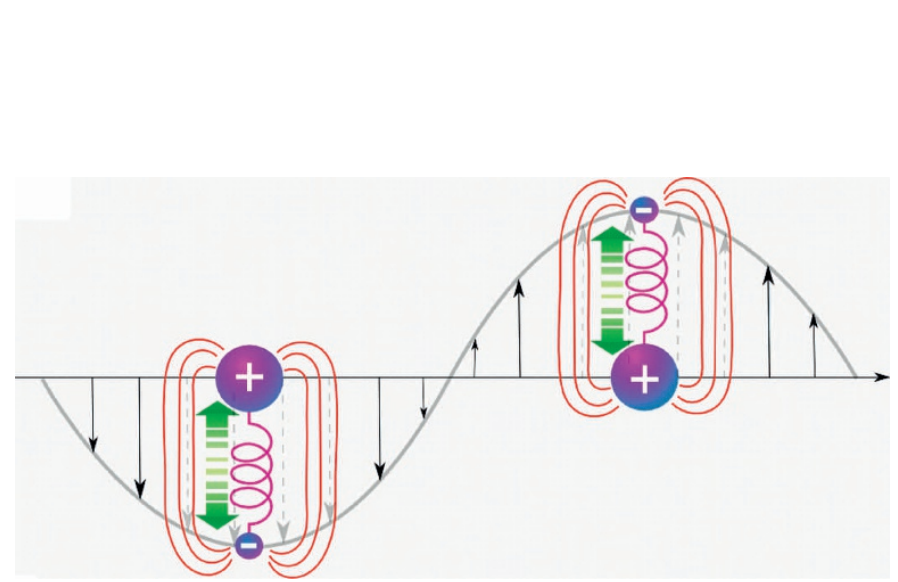

c

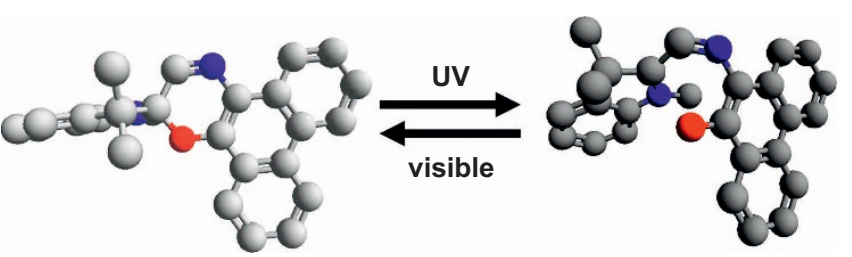

900; Waltham, Massachusetts, America). The measurements were made using glass as the background.

\section{RESULTS AND DISCUSSION}

When electromagnetic waves illuminate a dipole (i.e., atoms, molecules and nanoparticles) that is much smaller than their wavelength at the resonance frequency, the field is considered homogenous. This approximation, first introduced by Rayleigh ${ }^{8}$, is called a quasi-static approximation. Accordingly, the electric field inside of the dipole will drive the electron cloud relative to the positive core (Figure 1a). The attraction between the negatively charged electrons and the positive core ions generates a restoring force that causes oscillations. In the simplest case, the alternating surface charges form an oscillating dipole that radiates electromagnetic waves. At this point, the energy of light converts into the oscillation energy of the electronic cloud, where e

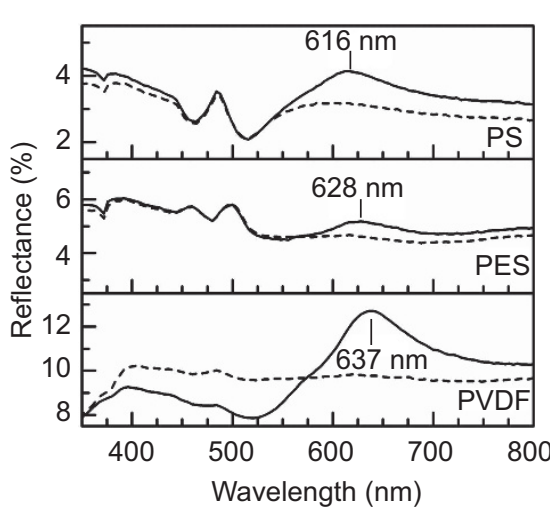

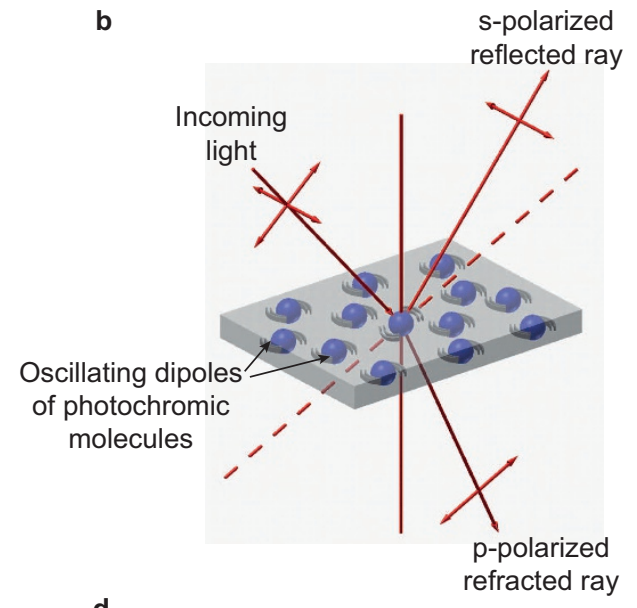

d

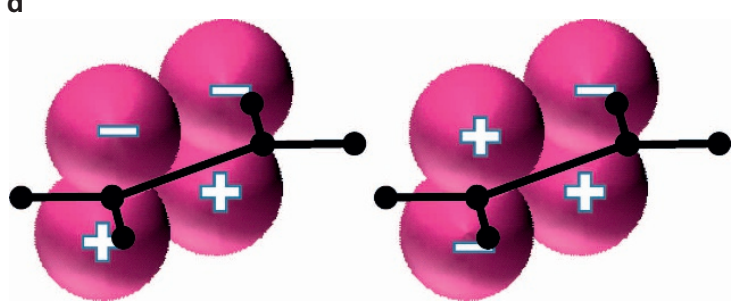
- After UV

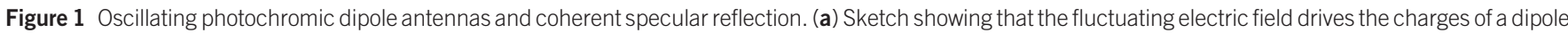

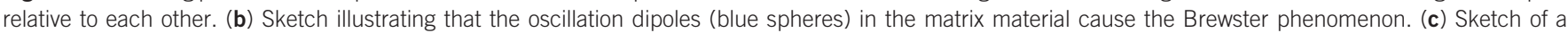

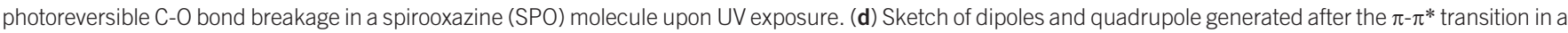

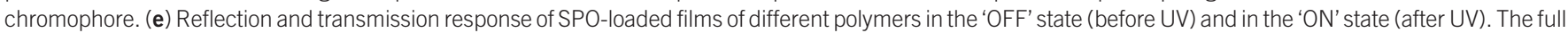
names of the polymer matrices can be observed in the Table 1. 
the dipole acts as an antenna that oscillates and radiates light to the far field. In the same way, an oscillating sheet of charges ${ }^{9}$ will generate electric and magnetic fields due only to the local change in the electric field that it in turn induces, for example, electric dipole and quadrupole moments, which eventually cause the reflection of light from objects ${ }^{10,11}$. Specular reflection is increased or diminished depending on the polarization of light and the angle of incidence as first demonstrated by É.-L. Malus in 1808 and further confirmed by D. Brewster in 1815. Indeed, this effect has been shown and discussed as purely geometrical, neglecting the illuminated matter enclosed ${ }^{12}$. These observations contradicts with the electromagnetic dipole radiation concept that is used to explain the phenomenon, as recognized by several physicists $^{13,14,15,16,17}$. However, while the origin of the phenomenon is not yet fully understood, we believe that the collective oscillation of dipoles governs a collective and mutual dipolar coupling even if the dipoles are randomly distributed. Here, we restrict ourselves to demonstrate the phenomenon experimentally, aiming to implement it for designing novel photoactive optical devices. For example, for the case of radiating dipoles (i.e., chromophores) embedded in a transparent polymeric matrix, a specular reflection and molecular Brewster phenomenon occurs, controlled by the oscillating dipoles of the molecules (Figure 1b). In this context, whenever the electric dipoles oscillate perpendicular to the electric field's direction of propagation, there will be no radiation reflected back into the first medium. Therefore, a reflection minimum for the p-polarized wave exists at a certain grazing angle, which gives rise to the Brewster phenomenon ${ }^{11}$. Because it is controlled on the molecular level, a switching in molecular behavior should effect both the reflection and Brewster phenomenon. Thus, by using chromophores, the Brewster phenomenon would be solely controlled by dynamic light switching and the molar polarizability of the dipole as recognized by Clausius-Mossotti. It is well-known that the molecular polarizability of the photochromic molecules changes upon illumination with UV light. However, in this context, the role of the surrounding medium in the work performed by Clausius-Mossotti is described as homogeneous with constant dielectric (i.e., refractive index). While this picture is macroscopically acceptable for nonpolar medium, it does not fit for the case of polar medium possessing a net dipole moment. Here, there is a mutual coupling between the dipole antenna generated by excitation and the permanent dipole of the surrounding matrix. According to the generated dipolar coupling, a mutual polarization is extrinsically and anisotropically raised and hence governs the optical response. Based on this concept, molecular Brewster wavelength will be introduced here. In other words, while the Brewster angle is determined by the refractive index at the interface, the Brewster wavelength is determined by the mutual dipolar coupling of the surrounding matrix and the matter enclosed. Thus, surrounding matrices that have little difference in refractive index (similar Brewster angles) can be easily distinguished based on the dipole moment (i.e., polarity) of their pristine matrix (Brewster dip at different wavelengths).

To confirm our hypothesis, we used a photoswitchable compound blended in transparent polymeric matrices and found that specular

Table 1. Refractive indices and dipole moments of the polymer matrices.

Polymer Real part of refractive index $(n)$ Dipole moment $(D)$

Polystyrene (PS)

Polyethersulfone (PES)

Polyvinylidenefluoride (PVDF)

$\begin{array}{cc}1.58-1.61^{[13]} & 0.2-0.3^{[14]} \\ 1.55-1.67^{[15]} & 1.62^{[16]} \\ 1.42^{[17]} & 2.1^{[18]}\end{array}$

reflection and Brewster phenomenon are controlled by UV light illumination. The photochromic system was fabricated by spin coating 50-nm films of different polymers doped with SPO onto glass substrates. The polymers used for spin coating are listed in the Table 1, along with their respective refractive index and dipole moment. The matrices were selected such that there was only a slight difference in their refractive index but a significant difference in their dipole moments, enabling our concept of Brewster wavelength to be tested.

A schematic illustration of the molecular structure of the spirooxazine (SPO) that was used in this work is shown in Figure 1c. SPO belongs to the family of molecules displaying photochromic properties. SPO in the 'closed form/OFF' state has absorption only in the ultraviolet (UV) spectral region. Upon illumination with UV radiation, the $\mathrm{C}-\mathrm{O}$ bond of the closed form breaks, and the molecule transforms to its planar merocyanine state (or 'open form/ON state'). Owing to its planar structure and extended $\pi$-conjugation, merocyanine shows a delocalized transition in absorption shifted to the visible region, which originates from the promotion of an electron from the bonding level $(\pi)$ to the antibonding level $\left(\pi^{*}\right)$ under UV illumination $^{5}$. Figure $1 \mathrm{~d}$ shows the typical dipole and quadruple nature of the oscillating chromophores originating from the $\pi-\pi^{*} \operatorname{transition}^{18}$. The spirooxazine used in this work showed a pronounced response in reflection at approximately $600 \mathrm{~nm}$ in addition to its well-known absorption after exposure to UV light (Figure 1e). The photochromic molecules can be switched back to their original closed form by illumination with white light, giving them the ability to be dynamically controlled by light.

To confirm our postulation of coherent coupling in the system and to gain a deeper understanding of the optical response, the specular reflection properties of the films using $s-(R s)$ and p-polarized (Rp) light under oblique incidence were investigated. As shown in Figure 2, there was no resonance reflection peak in the visible range before UV illumination, irrespective of the polarization. However, upon exposure to UV light, a resonance reflection peak appeared at approximately $600 \mathrm{~nm}$. It is well recognized that dipole radiation at an interface differs significantly for $\mathrm{p}$ - and s-polarized waves ${ }^{11}$, and as a result, Rp- and Rsshowed quite different intensities. Under s-polarized light, the oscillation direction of electric dipoles is always parallel to the oscillation direction of the reflected light; hence, Rs increased gradually with increasing incidence angle ${ }^{11}$. A similar trend was observed for p-polarized light; however, at certain angles where the dipole moments were parallel to the direction of the reflected light, a dip appeared in the intensity of light reflection ${ }^{11}$. Although the reflection did not vanish completely, owing to the molecules' absorption, there was a minimum value at the same critical incident angle of $65^{\circ}$ for all polymer matrices. Because the reflection peak/dip appeared/disappeared dynamically upon UV/white light illumination, the current finding is an important step toward designing novel photochromic devices.

Having introduced and proven photoswitchable specular reflection in glassy dipolar composite medium, we considered examining polarity sensing using the unpolarized/polarized light reflection while introducing the Brewster wavelength sensor, first with p-polarized light. Figure $3 \mathrm{a}$ shows the comparison of the reflection spectra at oblique angles of $45^{\circ}$ and $65^{\circ}$ (at the Brewster dip) in a film of 30 wt-\% SPO embedded in nonpolar PS and polar PVDF matrices. It is obvious that these reflection peaks/dips are red-shifted based on the dipole moments (Table 1$)^{19-26}$. While the reflection peaks (at $45^{\circ}$ incidence) showed a spectral shift of $11.1 \mathrm{~nm}$, the reflection dip that occurred at the Brewster condition (i.e., at $65^{\circ}$ incidence) showed a hugely amplified spectral shift of $40 \mathrm{~nm}$. To the best of our knowledge, 

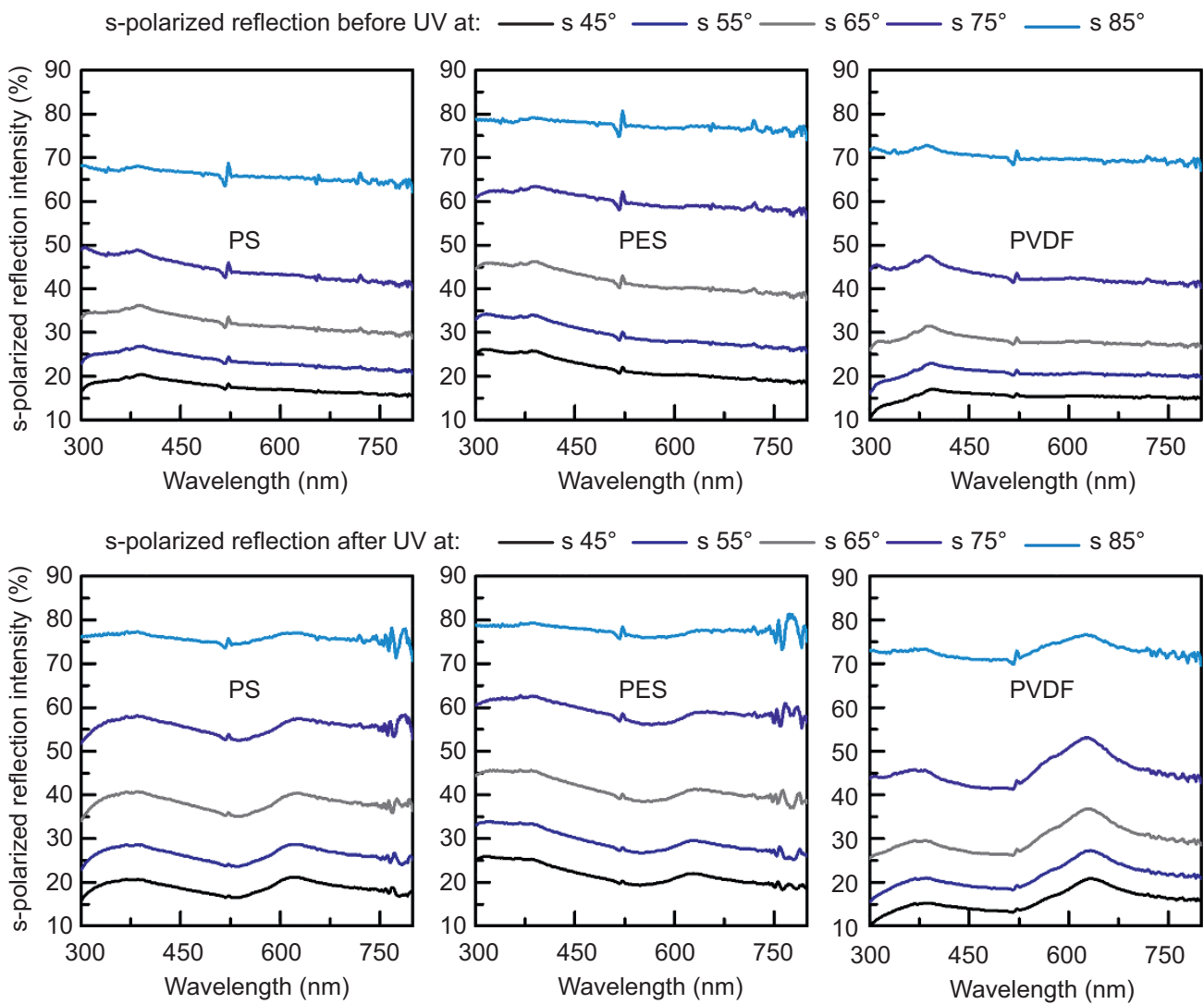

p-polarized reflection before UV at:

$\mathrm{p} 55^{\circ}$
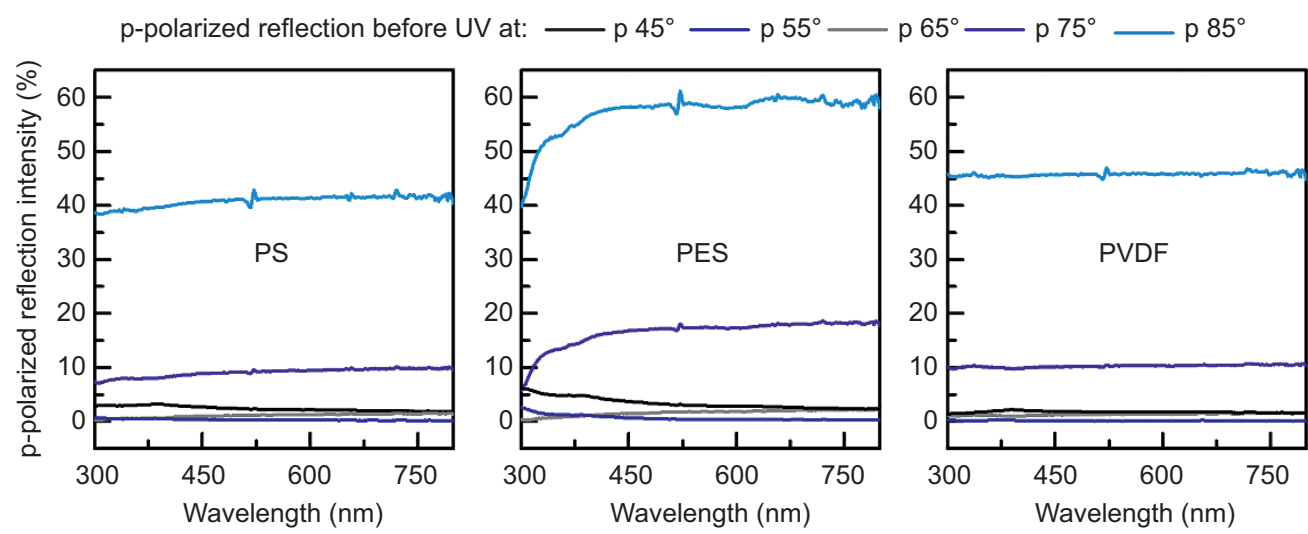

p-polarized reflection after UV at:
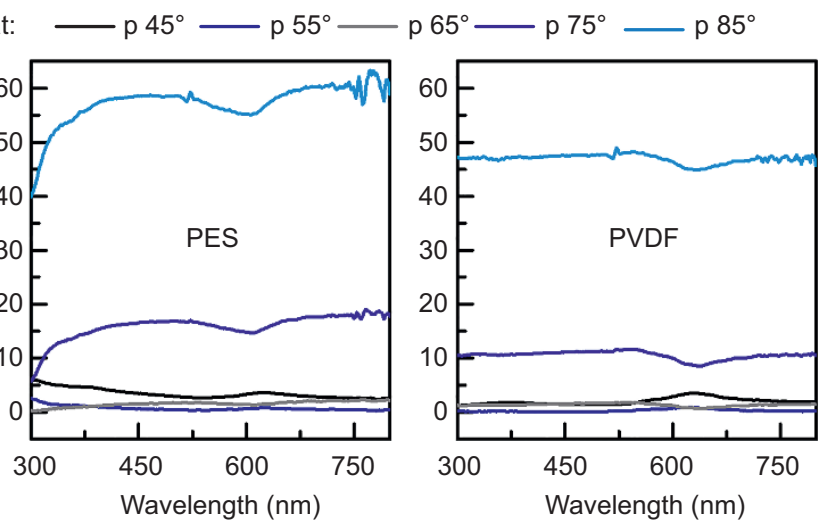

Figure 2 Specular reflection and ellipsometric analysis. Angular reflectance of p-and s-polarized light from films composed of SPO embedded in three different polymeric matrices before and after exposure to UV light. 
a

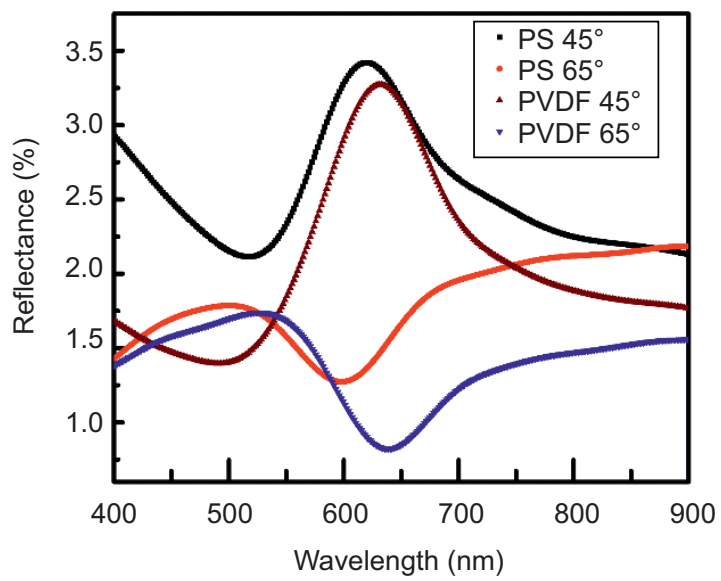

C

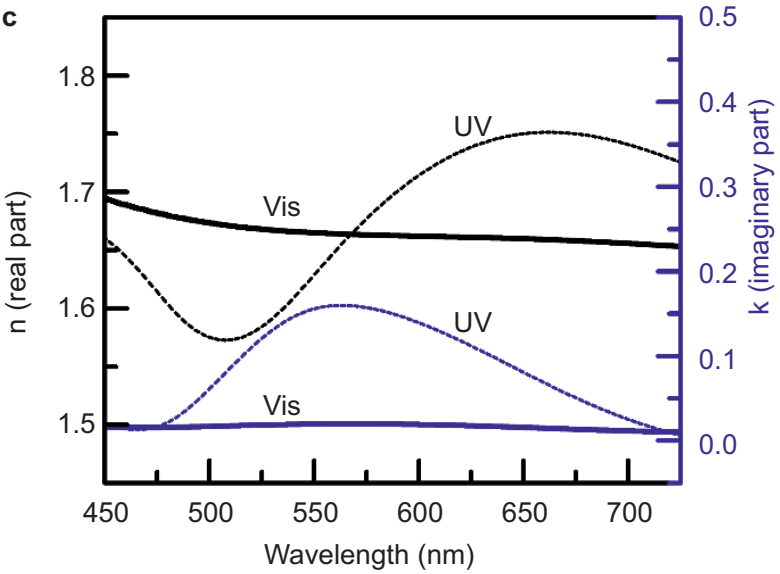

b

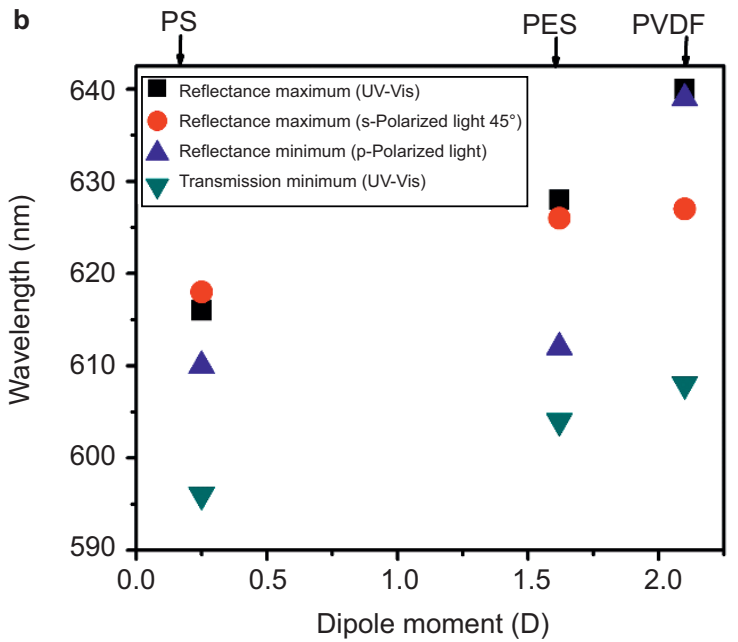

d

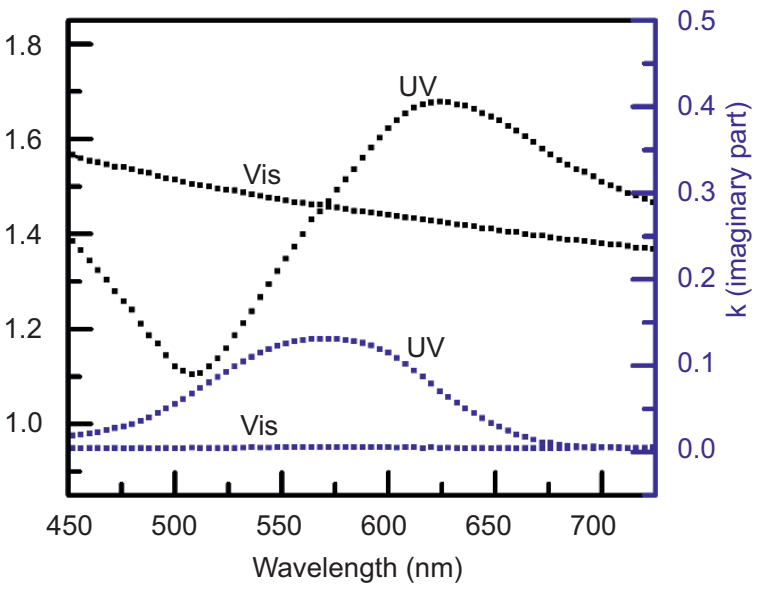

Figure 3 The Brewster wavelength for matrix polarity sensing and the role of reflection in the refractive index modulation. (a) Ellipsometry results of UV light-exposed samples showing a significant, matrix dependent change in the Brewster wavelength, observed at the Brewster angle of $65^{\circ}$ (p-polarized light). (b) UV-Vis and ellipsometry results showing an increase in reflectance maxima with an increasing dipole moment of matrix polymers surrounding the SPO molecules. (c, $\mathbf{d}$ ) Real and imaginary parts of the refractive index of PS-SPO film under Vis/UV illumination: (c) using ellipsometry results; (d) numerical simulation.

this demonstrates the highest sensitivity of an optical sensor toward matrix polarity and proves our concept of Brewster wavelength. It is notable that the shift in Brewster wavelength seems to be more affected by the dipole moment than by the real part of the refractive index that is nearly the same for both the polymers, indicating the role of dipolar interactions. Nevertheless, more investigations are needed to understand the Brewster wavelength phenomenon. The difference between the absorption/reflection-based polarity determinations is illustrated in Figure $3 \mathrm{~b}$, showing the comparison of the transmission and reflection maxima and minima from SPO films in different polymers, plotted against the dipole moment of each polymer. These measurements were made under normal incidence as well as grazing incidence at an incident angle of $45^{\circ}$ and $65^{\circ}$ (i.e., the Brewster angle). For normal incidence, the sensitivities were determined from a linear fit and show a change of $6.3 \mathrm{~nm} /$ Debye for the transmission measurements and $12 \mathrm{~nm} /$ Debye for the reflection measurements. Indeed, while the reflection measurements can provide clear spectral differences between the polymer matrices, especially the polar matrices, the transmission technique was not sensitive enough to provide clear spectral differences, as shown in Figure 1e. However, under grazing incidence, nonlinear behaviors are observed, indicating an extreme sensitivity to matrix polarity. It is worth mentioning that the sensitivity based on the shift in reflection peak maxima was similar to that obtained while using s- and p-polarized light but was less than the sensitivity based on the Brewster reflection dip. Thus, a novel analytical platform wherein the polarity of the host medium can be identified and photo-detected with high accuracy using reflection measurements, especially at the Brewster wavelength, has been demonstrated. Indeed, a stable photoswitchable system is required for practical application. It is observed that the photo recovery of one cycle is clearly independent of the polymer type (Supplementary information Fig. S1), while the long-term stability is highly dependent on the glass temperature of the polymeric host. For example, Supplementary information Fig. S2 shows that PS is more stable to switching fatigue than PVDF, owing to the low glass temperature of PVDF polymer that corresponds to the enhanced diffusion of the molecules. The system based on a softer matrix (PVDF has a glass temperature of only $-30{ }^{\circ} \mathrm{C}$ compared to $100{ }^{\circ} \mathrm{C}$ for PS) is much less long-lasting.

The photoswitchable reflection by photochromic molecules randomly embedded in a polymeric matrix demonstrated here deepens our understanding of refractive index modulation, which is to date solely inferred from the molecular absorption ${ }^{27-32}$; these insights could facilitate the development of a novel strategy for interferencebased optical devices under UV illumination. 
a
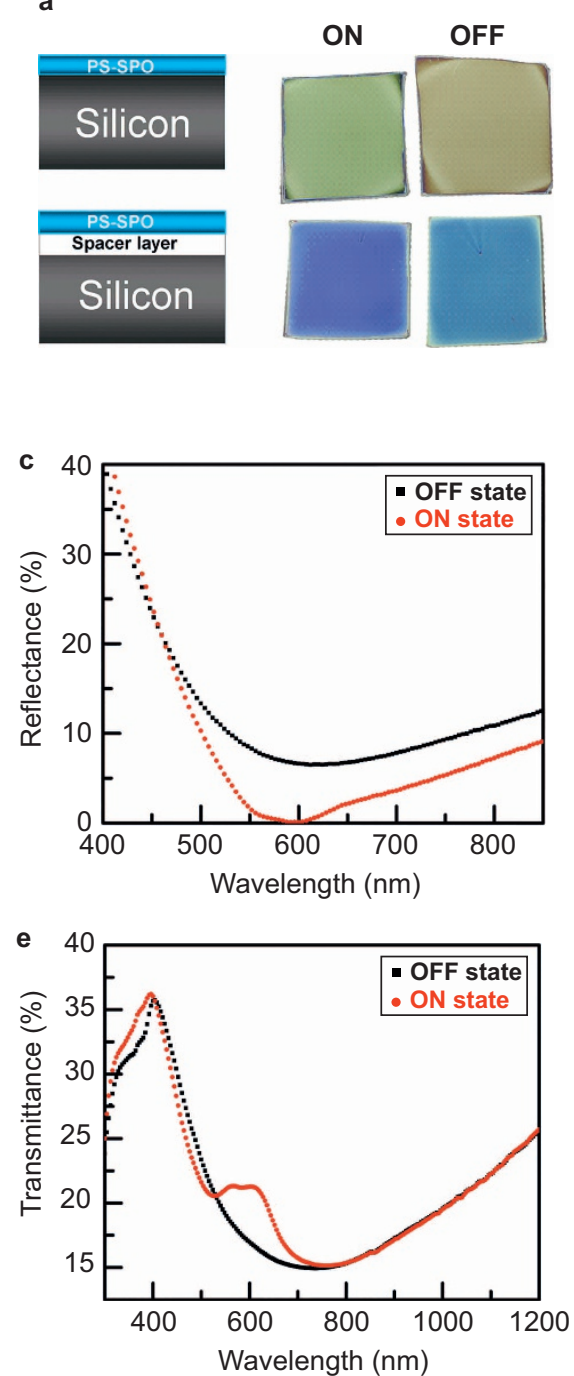

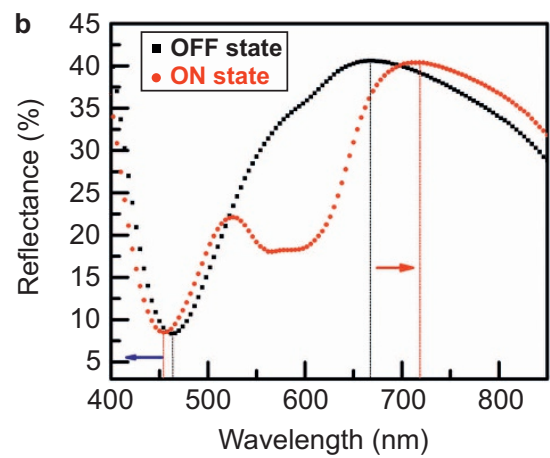

d

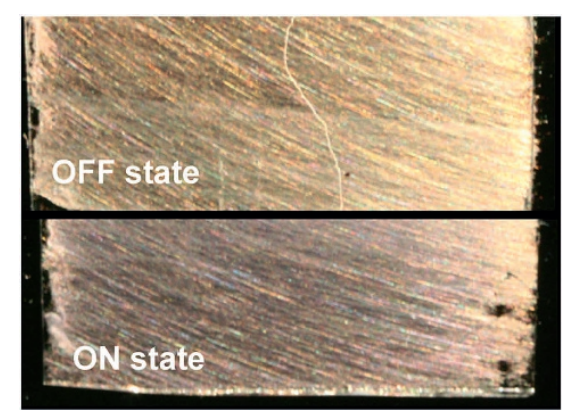

f

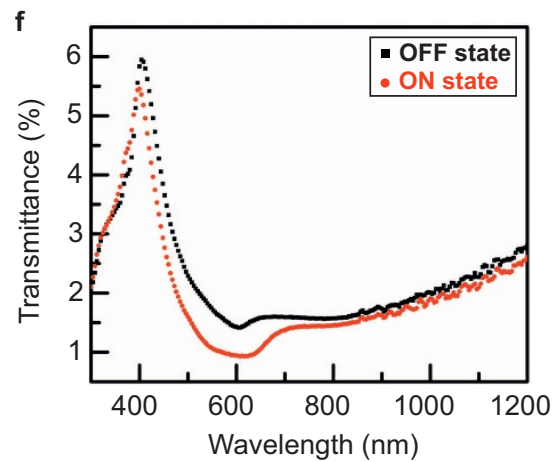

Figure 4 Color and transparency by tailored molecular photonic coupling. (a) Effect of a thin spacer layer of silicon oxide between the 'PS-SPO' film and silicon wafer on the reflected color for the 'OFF/before UV' and 'ON/after UV' states. (b) UV-Vis reflectance spectra of a PS-SPO film coated on a silicon wafer; 'OFF/before UV' state, black line and 'ON/after UV' state, red line. (c) UV-Vis spectra of a PS-SPO film coated on a silicon wafer with a silicon oxide spacer layer; ('OFF/before UV' state, black) and ('ON/after UV' state, red). (d) Photo of a part of the fibers in the 'OFF/before UV' state and in the 'ON/after UV' state, as observed with transmitted light. (e, f) UV-Vis spectra of PVDF-SPO fibers of different thicknesses ('OFF/before UV' state, black) and ('ON/after UV' state, red): (e) $950 \mathrm{~nm}$ thick fibers; (f) 750 nm thick fibers.

Figure $3 \mathrm{c}$ shows a typical light modulated refractive index of $\sim 20 \%-30 \%$ SPO photochromic molecules embedded in a PS matrix before and after exposure to UV light, as measured by ellipsometry. While the imaginary part, which is attributed to molecular absorption, showed a peak at $560 \mathrm{~nm}$, the real part of the refractive index underwent an anomalous dispersion that peaked at $660 \mathrm{~nm}$.

We stress here that the dispersive curve of the real part is controlled by the dynamic reflection of the photochromic molecules. We further confirm it by calculating the refractive index modulation from the absorption and reflection coefficients by direct numerical simulations. The results of calculations are shown in Figure 3d. For the numerical calculations, the index of refraction was restored with the following relation $^{33}$ :

$$
n=\frac{1+R}{1-R}+\sqrt{\frac{4 R}{(1-R)^{2}}-k^{2}}
$$

where $k$ is given by;

$$
k=\frac{\lambda}{4 \pi} \times \frac{A}{d}
$$

Here, $A$ is the normalized absorption coefficient, $R$ is the normalized reflection coefficient, $\lambda$ is the wavelength and $d$ is the thickness. It is evident that the results are quite similar, with minor differences owing to the difference in the adopted methods.

Most photochromic devices presented to date are based on the modulation of the imaginary part of the refractive index ${ }^{27-32}$. However, we believe that our findings will change the picture, and novel optical devices can be designed by bringing together reflection, inferences and refractive index modulation while tailoring and engineering the coupling. To highlight our findings without going into too much detail, we give two examples, namely, tailored coloration and photonic wavelength shift of a reflective substrate coated with a 
'photochromic film' and a new class of optically active photoswitchable photonic transparency using photochromic fibers.

To explore the role of refractive index modulation (real/imaginary) and consequently manifest our findings of photoswitching reflection, we spin coated PS-SPO film on silicon substrate with and without a spacer layer of silicon oxide. Figure 4 a shows the sketch of the coating arrangement along with the different coloration of the coating before and after UV illumination. The UV-Vis analysis of the system wherein the photochromic film was directly coated on the silicon substrate is shown in Figure 4b. It can be observed that the film was brownish (Figure 4a) in the 'closed/OFF' state and had the reflection maximum and minimum at approximately $665 \mathrm{~nm}$ and $462 \mathrm{~nm}$, respectively (Figure $4 \mathrm{~b}$ ), owing to constructive and destructive interference.

However, once illuminated by UV light, a reflection dip appeared at approximately $604 \mathrm{~nm}$ and the color changed to green (Figure $4 \mathrm{a}, 4 \mathrm{~b}$ ). Moreover, the reflection peak underwent a pronounced red shift of more than $50 \mathrm{~nm}$, while the reflection dip blue shifts by approximately $12 \mathrm{~nm}$, owing to the modulation of the real part of the refractive index. When a spacer layer of $50 \mathrm{~nm}$ of silicon oxide between the PS-SPO film and silicon substrate was introduced, the film color became light blue without UV exposure and changed to dark blue after UV exposure (Figure 4a). Figure 4c shows the UV-Vis analysis of this system where a broad reflection minimum occurred at approximately $620 \mathrm{~nm}$. Upon illumination with UV light, we observed a drop in reflection along with a slightly blue shifted reflection minimum at approximately $600 \mathrm{~nm}$, i.e., at the wavelength where absorption of photochromic molecules takes place (Figure 4c, 1e). No splitting was observed as the reflection minimum was indeed in the range where the imaginary part of the refractive index of the photochromic film was dominant. This result highlights the control of color and peak shifts, based on the interference and refractive index of the photochromic film, and introduces a very promising design map for controlling the optical properties of photochromic devices based on tailoring molecular photonic coupling instead of plasmonic coupling shown in our previous reports ${ }^{5,6}$.

To support our finding of controlling the optical properties of a photoactive device and to introduce the concept of photochromic transparency, we designed another photochromic system based on nanofibers.

Photochromic fibers were fabricated by the electrospinning technique from a blend of polymer solution and SPO. A typical image of fibers is shown in Figure 4d. Electrospinning allowed us to control the fiber diameter. Thus, we were able to tune the resonance peak of fibers to coincide with the absorption resonance of molecules or to overlap with the peak of the real part of their refractive index (i.e., the reflection dominant region). Figure $4 \mathrm{e}$, $4 \mathrm{f}$ shows this effect using PVDF fibers with an average diameter, $d$, of $\sim 950 \mathrm{~nm}$ and $\sim 750 \mathrm{~nm}$, which contained $20-30 \mathrm{wt}-\%$ SPO, and possessed a broad resonance peak centered at $720 \mathrm{~nm}$ and $620 \mathrm{~nm}$, respectively. After exposure to UV light, the position of the resonance peak shifted, and the optical response of the system changed with varying fiber size.

The UV-Vis analysis in the transmission mode of optically active PVDF fibers of coarser diameter $(\sim 950 \mathrm{~nm})$ is shown in Figure $4 \mathrm{e}$. The fibers have a transmission minimum at approximately $720 \mathrm{~nm}$ when the embedded chromophore was in the 'closed/OFF' state. Once illuminated with UV light, an enhancement in transmission appeared at approximately $600 \mathrm{~nm}$, i.e., at the wavelength where absorption of photochromic molecules was expected. Even more pronounced was the resonance peak splitting, revealing two separate peaks at 568 and $604 \mathrm{~nm}$. Such splitting allowed us to demonstrate a molecular photonic coupling, analogous to molecular plasmonic coupling ${ }^{30,34-36}$. However, in the case of finer fibers, photo absorption was observed after exposure to UV light (Figure 4f), which is generally expected from SPO molecules inside of polymer films or solvent environments. For fine fibers, neither an increase in transmission nor splitting was observed, and the system behaved similarly to the silicon/spacer system (Figure 4c) because both were controlled mainly by the imaginary part of the refractive index. Thus, we show a new strategy for controlling the molecular coupling in an optically active system while tailoring the type of coupling in terms of weak and strong.

\section{CONCLUSIONS}

Taken together, we demonstrated the first experimental evidence of specular reflection and Brewster phenomenon of chromophores, enabling the design of novel optically active systems. Our experimental findings need further investigation in terms of numerical simulation to fully understand the phenomenon. Yet, we believe that our work leverages our comprehension of several optical phenomena based on the photoswitchable molecules and enables the design of new lightdriven functional devices. For example, while the common wisdom that changes in the optical properties of the photoswitchable molecules is mainly due to electronic transitions, here, we stressed the role of electronic polarization and dipole radiation as main handles for tailoring optical properties. It turns out that the collective oscillations of the excited molecules give rise to the collective and coherent coupling of randomly distributed molecular dipoles. In this context, the coupling is not only affected by the polarizability of the molecules and the macroscopic refractive index of the surrounding, but it is highly related to the dipole moment of the surrounding medium and the dipolar interactions in the case of polar matrices. Furthermore, the dispersive curve of the real part is controlled by the dynamic reflection of the photochromic molecules. We confirm our hypothesis by calculating the refractive index modulation from the absorption and reflection coefficients by direct numerical simulations. We successfully demonstrated the concept of the Brewster wavelength and introduced the concept of 'tailored molecular photonic coupling' for the design of optically active media. From a technological point of view, the uniqueness of our results rely on the introduction of a new concept for highly localized sensing of matrix polarity and tailoring of the optical and photonic properties of several devices by adjusting the photonic response of the medium with the real and imaginary parts of the refractive index of the photoswitchable molecules in the 'ON' state. That allowed us to demonstrate and tailor the coupling in terms of weak molecular photonic coupling (when the photonic resonance of the host/nearby medium is coincident with the imaginary part of the photoswitched molecules) and strong molecular photonic coupling and splitting (when the photonic resonance is coincident with the real part of the photoswitched molecules). We demonstrate the validity of our concept with two different systems (film and fibers). To our knowledge, this method of engineering coupling is reported for the first time in this paper. We strongly believe that our finding changes the fundamental understanding of coherent coupling in general, especially in disordered systems, and will open a new area of optics research.

\section{ACKNOWLEDGEMENTS}

We gratefully acknowledge financial support from the German Research Foundation (DFG) through SFB677 (C09) and partial financial support from SFB677 (C01) as well as the German Academic Exchange Service (DAAD). ME thanks the Initiative and Networking Fund of the Helmholtz Association (Grant no. VH-NG-523) for providing the financial support to start his 
research group. We also would like to thank Z Kareh for her contribution in the fatigue measurements and S Rehders for drawing the schematics.

1 Russew MM, Hecht S. Photoswitches: from molecules to materials. Adv Mater 2010; 22: 3348-3360

2 Irie M. Photochromism: memories and switches-introduction. Chem Rev 2000; 100 : 1683-1684.

3 Bechinger C, Ferrere S, Zaban A, Sprague J, Gregg B. Photoelectrochromic windows and displays. Nature 1996; 383: 608-610.

4 Grinthal A, Aizenberg J. Adaptive all the way down: building responsive materials from hierarchies of chemomechanical feedback. Chem Soc Rev 2013; 42: 7072-7085.

5 Jamali M, Hedayati MK, Mozooni B, Javaherirahim M, Abdelaziz R et al. Photoresponsive transparent conductive metal with a photobleaching nose. Adv Mater 2011; 23: 4243-4247.

6 Hedayati MK, Javaheri M, Zillohu AU, El-Khozondar HJ, Bawa'aneh MSLA et al. Photo-driven super absorber as an active metamaterial with a tunable molecularplasmonic coupling. Adv Opt Mater 2014; 2: 705-710.

7 Klajn R. Spiropyran-based dynamic materials. Chem Soc Rev 2013; 43: 148-184.

8 Rayleigh L. Theory of reflection by opaque bodies. Phil Mag 42: 441.

9 Kittel C, Purcell E. Berkeley Physics Course. New York, USA: McGraw-Hill; 1966.

10 Martin S. An Introduction to Ocean Remote Sensing. New York, USA: Cambridge University Press; 2004.

11 Fujiwara H. Spectroscopic Ellipsometry: Principles and Applications. Tokyo, Japan: John Wiley \& Sons; 2007.

12 Biot MA. Some new aspects of the reflection of electromagnetic waves on a rough surface. J Appl Phys 2004; 28: 1455-1463.

13 Kerker M. The Scattering of Light, and other Electromagnetic Radiation. New York, USA: Academic Press; 1969.

14 Movchan AB, Poulton CG, Botten LC, Nicorovici NA, McPhedran RC. Noncommuting limits in electromagnetic scattering: asymptotic analysis for an array of highly conducting inclusions. SIAM J Appl Math 2001; 61; 1706-1730.

15 Biot MA. Generalized boundary condition for multiple scatter in acoustic reflection. J Acoust Soc Am 1968; 44: 1616-1622.

16 Stratton J. Electromagnetic Theory. New York, USA: John Wiley \& Sons; 2007.

17 Bohren C, Huffman D. Absorption and Scattering of Light by Small Particles. New York, USA: John Wiley \& Sons; 2008

18 Atkins PW, Friedman RS. Molecular Quantum Mechanics. New York, USA: Oxford University Press; 2011.

19 Kasarova SN, Sultanova NG, Ivanov CD, Nikolov ID. Analysis of the dispersion of optical plastic materials. Opt Mater 2007; 29: 1481-1490.

20 Mark HF. Encyclopedia of Polymer Science and Technology: Step-reaction Polymerization to Thermoforming. New Jersey, USA: Interscience Publishers; 1970.
21 Sen A, Kandpal L. Proceedings of Recent Advances in Polymers and Composites. New Delhi, India: Allied Publishers; 2000.

22 Margolis JM. Engineering Thermoplastics-properties and Applications. New York, USA: Taylor \& Francis; 1985

23 Biron M. Thermoplastics and Thermoplastic Composites: Technical Information for Plastics Users. Oxford, UK: Elsevier; 2012.

24 Mark HF. Encyclopedia of Polymer Science and Technology: Molding to Petroleum Resins. New Jersey, USA: Interscience Publishers; 1968.

25 Nussbaumer RJ, Caseri WR, Smith P. Reversible photochromic properties of $\mathrm{TiO}_{2}$ polymer nanocomposites. J Nanosci Nanotechnol 2006; 6: 459-463.

26 Zhang QM, Bharti V, Kavarnos G. Poly (vinylidene fluoride). (PVDF) and its copolymers. In: Encyclopedia of Smart Materials. Mel Schwartz (Ed.). New York, USA: John Wiley \& Sons; 2002.

27 Biteau J, Chaput F, Lahlil K, Boilot JP, Tsivgoulis G et al. Large and stable refractive index change in photochromic hybrid materials. Chem Mater 1998; 10: 1945-1950.

28 Kim EY, Choi YK, Lee MH. Photoinduced refractive index change of a photochromic diarylethene polymer. Macromolecules 1999; 32: 4855-4860.

29 Bertarelli C, Bianco A, D'Amore F, Gallazzi MC, Zerbi G. Effect of substitution on the change of refractive index in dithienylethenes: an ellipsometric study. Adv Funct Mater 2004; 14: 357-363.

30 Dintinger J, Klein S, Ebbesen TW. Molecule-surface plasmon interactions in hole arrays: enhanced absorption, refractive index changes, and all-optical switching. Adv Mater 2006; 18: 1267-1270.

31 Zheng YB, Yang YW, Jensen L, Fang L, Juluri BK et al. Active molecular plasmonics: controlling plasmon resonances with molecular switches. Nano Lett 2009; 9: 819.

32 Zheng YB, Kiraly B, Cheunkar S, Huang TJ, Weiss PS. Incident-angle-modulated molecular plasmonic switches: a case of weak exciton-plasmon coupling. Nano Lett 2011; 11: 2061-2065.

33 Pankove J. Optical Processes in Semiconductors. New York, USA: Dover Publications; 2012.

34 Dulić D, van der Molen S, Kudernac T, Jonkman HT, De Jong JD et al. One-way optoelectronic switching of photochromic molecules on gold. Phys Rev Lett 2003; 91: 207402.

35 Dintinger J, Robel I, Kamat PV, Genet C, Ebbesen TW. Terahertz all-optical moleculeplasmon modulation. Adv Mater 2006; 18: 1645-1648.

36 van der Molen SJ, Liao J, Kudernac T, Agustsson JS, Bernard L et al. Light-controlled conductance switching of ordered metal-molecule-metal devices. Nano Lett 2009; 9: $76-80$.

(c) (i) $\odot$ This license allows readers to copy, distribute and transmit the

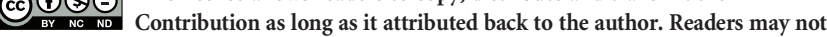
alter, transform or build upon the Contribution, or use the article for commercial purposes. Please read the full license for further details at - http://creativecommons.org/licenses/ by-nc-nd/4.0/ 